\title{
SPIN WAVES AND TEMPERATURE DEPENDENCE OF MAGNETIZATION IN NICKEL THIN FILMS COVERED WITH IRON AND COPPER
}

\author{
R. ŚWIRKOWICZ \\ Institute of Physics, Warsaw University of Technology. \\ Koszykowa 75, 00-662 Warszawa, Poland \\ (Received April 30, 1993; in final form May 31, 1993)
}

\begin{abstract}
Energy spectrum and amplitudes of spin waves, as well as the temperature dependence of the local magnetization in Ni thin films covered with $\mathrm{Fe}$ and $\mathrm{Cu}$ overlayers are investigated within the framework of the itinerant electron approach. Two acoustic surface modes strongly localized in Fe overlayers are found. Energies of these modes are very close to each other but they are considerably lower than in Ni films with free surfaces. The presence of the strongly localized low-energy modes leads to fast decrease in the surface magnetization as compared to the central layer one. The quasi-linear temperature dependence of the surface magnetization is found whereas the central layer magnetization follows a quasi $T^{3 / 2}$ law. On the other hand, in films covered with $\mathrm{Cu}$ the temperature dependence of the magnetization can be fitted to the Bloch law for all layers. Inside the film the Bloch coefficient weakly depends on the layer index but it rapidly decreases at the surface in the $\mathrm{Cu}$ layers.
\end{abstract}

PACS numbers: 75.10.Lp, 75.30.Ds, 75.70.-i

\section{Introduction}

Recently, spin waves and temperature dependence of the local magnetization in the spin-wave regime have been investigated within the framework of the itinerant electron approach for semi-infinite systems [1,2], as well as for ultrathin films [3]. The detailed calculations have been performed mainly for films with free surfaces. However, the experimental [4] and theoretical [2] investigations show that the temperature dependence of the surface magnetization strongly depends on the surface conditions. In many systems the magnetization at the surface follows the Bloch $T^{3 / 2}$ law but the ratio of the Bloch coefficient at the surface to that in the bulk can depend on a sample $[5,6]$. The quasi-linear dependence is also observed [7]. It was pointed out that changes of the exchange coupling near the 
surface strongly influence the temperature dependence of the surface magnetization [2]. Softening of the surface exchange can cause a considerable increase in the prefactor $B_{\mathbf{s}}$ at the surface. Quasi-linear temperature dependence of the surface magnetization is possible in the case of strong softening.

Therefore, it seems interesting to investigate spin waves and their influence on the temperature dependence of the magnetization in systems with various surface conditions. Some attempts of describing such systems have already been undertaken. In Ref. [8] some model systems have been considered. Changing one of the parameters near the surface we have found that the ratio of Bloch coeflicients in the surface and central layers can depend on the surface parameters. However, it seems to us that more realistic approach to the problem should be undertaken. Especially it is interesting to investigate the spin waves and their influence on the temperature dependence of the magnetization in nickel films covered with iron planes. According to Mathon and Almad [2] the strong influence of the iron overlayer can be expected in such systems because of considerable softening of the exchange coupling between iron and nickel planes.

In the present paper the spin waves characteristic of coated nickel films are investigated. Calculations are performed for films with two different kinds of overlayers. Namely, films covered with iron, as well as with copper are taken into account. In both cases the band structure and the ground-state properties are calculated. Then the influence of the overlayer on the spin-wave spectrum, the amplitudes and damping is investigated. The temperalure dependence of the local magnetization in these two systems is also discussed.

\section{The method}

The calculations are based on the multiband IIamiltonian

$$
\begin{aligned}
& H=\sum_{\nu j m \sigma} E_{\nu} c_{\nu j m \sigma}^{+} c_{\nu j m \sigma}+\sum_{\substack{\left(\nu j, \nu^{\prime} j^{\prime}\right) \\
m m^{\prime} \sigma}} H_{m m^{\prime}}^{\nu \nu^{\prime}}\left(j, j^{\prime}\right) c_{\nu j m \sigma}^{+} c_{\nu^{\prime} j^{\prime} m^{\prime} \sigma}^{+} \\
& +\frac{1}{2} \sum_{\substack{\nu j \sigma \sigma^{\prime} \\
m m^{\prime} m_{1} m_{1}^{\prime}}} U_{m m_{1}^{\prime} m_{1} m^{\prime}}^{\nu} c_{\nu j m \sigma}^{+} c_{\nu j m_{1}^{\prime} \sigma^{\prime}}^{+} c_{\nu j m_{1} \sigma^{\prime}} c_{\nu j m^{\prime} \sigma} .
\end{aligned}
$$

$c_{\nu j m \sigma}$ denotes here an annihilation operator of an electron with spin $\sigma$ in the orbital state $m$ at the lattice point $\nu j$. The five $d$-bands are only taken into account. The one-electron potential $E_{\nu}$ is assumed to be the same for all $d$-states. The Slater-Koster parametrization is used for the hopping integrals $H_{m m^{\prime}}^{\nu \nu^{\prime}}\left(j, j^{\prime}\right)$. The elements of matrix $U$ represent the effective intra-atomic interactions between electrons. The Coulomb and exchange integrals corresponding to the same and different orbital states are assumed to be of the same value.

The films of various thicknesses are considered. The surface of the film is assumed to be perpendicular to the [001] direction. Numerical calculations are performed for nickel films covered with iron and copper. In the first case the Slater-Koster parameters appropriate for the non-magnetic iron are chosen. The one-electron potential $E$ is assumed to be equal to $1.8 \mathrm{eV}$ in iron layers whereas 
it is equal to $0.0 \mathrm{eV}$ in nickel planes. Value of parameter $E$ is chosen in such a way so that the proper number of electrons is obtained for both metals. The parameter $U$ takes values $0.9 \mathrm{eV}$ and $1.0 \mathrm{eV}$ in iron and nickel layers, respectively $[9,10]$. In the case of nickel film covered with one copper layer the parameters are $E_{\mathrm{Cu}}=-1.8 \mathrm{eV}$ and $U_{\mathrm{Cu}}=1.0 \mathrm{eV}$ [11]. The Slater-Koster parameters appropriate for copper are taken in the surface layer. Changes of the lattice constant in the óverlayers are not taken into account.

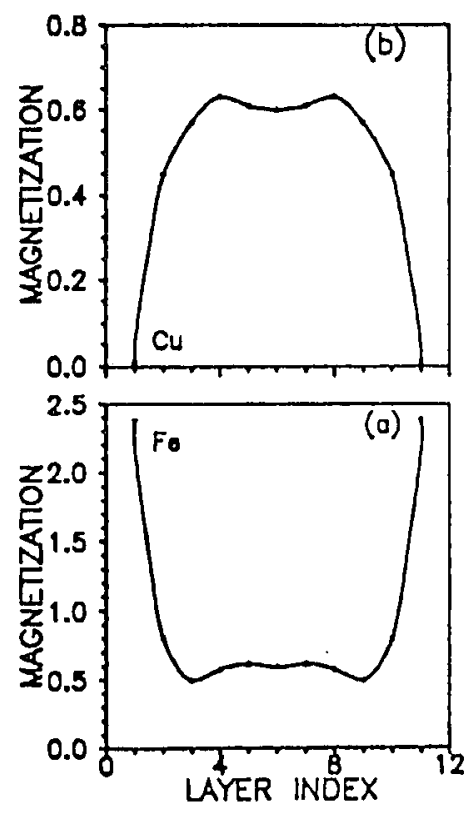

Fig. 1. The distribution of the magnetization in nickel films covered with (a) Fe and (b) $\mathrm{Cu}$.

The band structure and the one-electron ground-state properties characteristic of both systems are calculated. The dependence of the magnetic moment on a layer index for films covered with iron is presented in Fig. 1a. The considerable enhancement of the magnetization in layers closer to the surface is obtained. The surface magnetization is equal to 2.38 . Therefore, it is greater than in bulk iron but lower than the value obtained for the isolated $\mathrm{Fe}$ monolayer. The magnetization near the interface is strongly enhanced. It is a result of the $\mathrm{Fe}-\mathrm{Ni}$ interaction which leads to increase in minority spin holes. But the magnetic moment is suppressed in the subinterface $\mathrm{Ni}$ plane. Very small changes of the magnetization can be seen inside the film. In Fig. 1b the changes of the magnetization in films coated with copper are depicted. According to Fig. 1 it can be seen that the magnetic moment in the interface strongly depends on the environment. Whereas the moment is essentially enhanced in $\mathrm{Fe} / \mathrm{Ni}$ interface, il strongly drops in $\mathrm{Cu} / \mathrm{Ni}$. 
The results obtained here are qualitatively consistent with the ones calculated with the use of ab initio approach [12-14].

Spin waves which can propagate in the systems under consideration are investigated using the dynamic susceptibility method (see e.g. [3]). The susceptibility $\chi(q, E)$ is calculated within the framework of random phase approximation (RPA). The mixed Bloch-Wannier representation is used. Calculations are performed for various values of the two-dimensional vector $q$ which is assumed to be parallel to the surface. Spin-wave energies which correspond to poles of $\chi(q, E)$ are calculated from the equation

$$
\operatorname{det}\left|I-U \operatorname{Re} \chi^{0}(q, E)\right|=0 \text {, }
$$

where $\chi^{0}(q, E)$ denotes the susceptibility of non-interacting clectrons. The damping of the modes is determined from the imaginary part of $\chi$.

It should be pointed out that there are some problems connected with investigations of spin waves in pure bulk bcc Fe within the framework of the tight-binding approach [9]. The spin-wave energy appears to be strongly dependent on the band structure. The $s p$ - $d$ hybridization also plays an important role and the spin-wave stiffness constant can be negative if only $d$-bands are taken into account. However, according to [10] in iron monolayers it is possible to obtain positive spin-wave constant using only $d$-bands. Moreover, in the case of overlayers situated on Ni films there are modifications of the band structure and the density of electron states in nickel, as well as in iron planes, which are the results of the strong $\mathrm{Ni}-\mathrm{Fe}$ interaction (see Ref. [13]). More refined approaches are possible, however it seems reasonable to undertake the problem within the framework of the presented method. Such important effects as a hybridization of $d$-bands of $\mathrm{Ni}$ and Fe near the interface, which according to Ref. [13] strongly influcnce the one-electron results, can be taken into account within the framework of the assumed basis.

\section{Spin waves}

Amplitudes and energies of spin waves are calculated for various values of the wave vector $q$ for $\mathrm{Fe} / \mathrm{Ni} / \mathrm{Fe}$ and $\mathrm{Cu} / \mathrm{Ni} / \mathrm{Cu}$ systems. In the first case films which consist of $7,11,17$, and 23 monolayers (ML) are considered. It is found that in films covered with Fe the amplitudes of two modes, which correspond to the lowest energies, are very high in the surface Fe layers and quite small in nickel planes. Therefore, the highly localized acoustic surface modes are obtained in this case. On the other hand, the spin-wave modes, which correspond to higher energies, appear to be typical "bulk" modes. Their amplitudes are relatively high inside the film, whereas they are very low in the surface planes. The spin-wave amplitudes calculated for film consisting of $11 \mathrm{ML}$ are depicted in Fig. 2 for $q=(1,0)$ in $\pi / 4 a$ units, where $a$ denotes the lattice constant. In the region of small $q$ the amplitudes weakly depend on $q$. The localization of the surface modes slightly increases with $q$. However, for higher values of $q$ the amplitudes practically do not change. Quite different behaviour was found in the uncovered nickel films [15]. In such films a weakly localized acoustic surface mode was observed in the region of low $q$ values. For higher values of wave vector only modes of the "bulk" type were found. One 


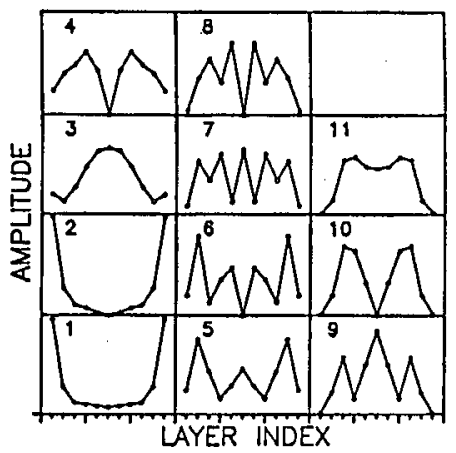

Fig. 2. The spin-wave amplitudes in $11 \mathrm{ML}$ film covered with Fe. The wave vector $q=(1,0)$ in $\pi / 4 a$ units.

can see therefore that the presence of the Fe overlayers leads to an appearance of highly localized surface modes, almost two-dimensional in their character. Such modes persist up to the Brillouin zone boundary. The result is in agreement with the one obtained within the framework of the IIeisenberg model, where it was found that in system with impurity planes the strongly localized modes appear near these planes $[16,17]$.

Dispersion relations calculated for films of various thicknesses covered with Fe are presented in Fig. 3. The curves obtained for modes which propagate in two different directions are depicted. Energies of the two acoustic surface modes are close to each other (they are degenerated or almost degenerated in the scale of the figure). It appears that for low $q$ values the energies of these modes slightly increase with the thickness of the film. On the other hand, energies of the low lying "bulk" modes decrease when the thickness of the film increases. Therefore, for thicker films the differences in energy of the "bulk" and surface modes are less distinct in the region of low $q$. For films consisting of $11 \mathrm{ML}$ the calculations are performed more accurately using greatcr numbcr of points in the irreducible part of the Brillouin zone. The dispersion curves are fitted to the $D q^{2}$ relation. It appears that the curves which correspond to the three low lying modes fit the relation quite well in the considerable part of the Brillouin zone. The fitting is much better than for uncovered nickel films. The stiffness parameter $D$ obtained for the first surface mode in uncovered films is over twice as high as the one found for films covered with $\mathrm{Fe}$. Therefore, the presence of Fe overlayers leads to considerable reduction of energy of the low lying modes. The result is understandable because the modes are mainly localized in Fe overlayers and lower spin-wave encrgy is expected in iron.

Spin waves which propagate in nickel films covered with Fe are well defined. Damping is very small and the modes with quite considerable energies can be well observed.

Next, the properties of spin waves are investigated in films covered with $\mathrm{Cu}$. The amplitudes of the modes for $q=(1,0)$ (in $\pi / 4 a$ units) are presented 


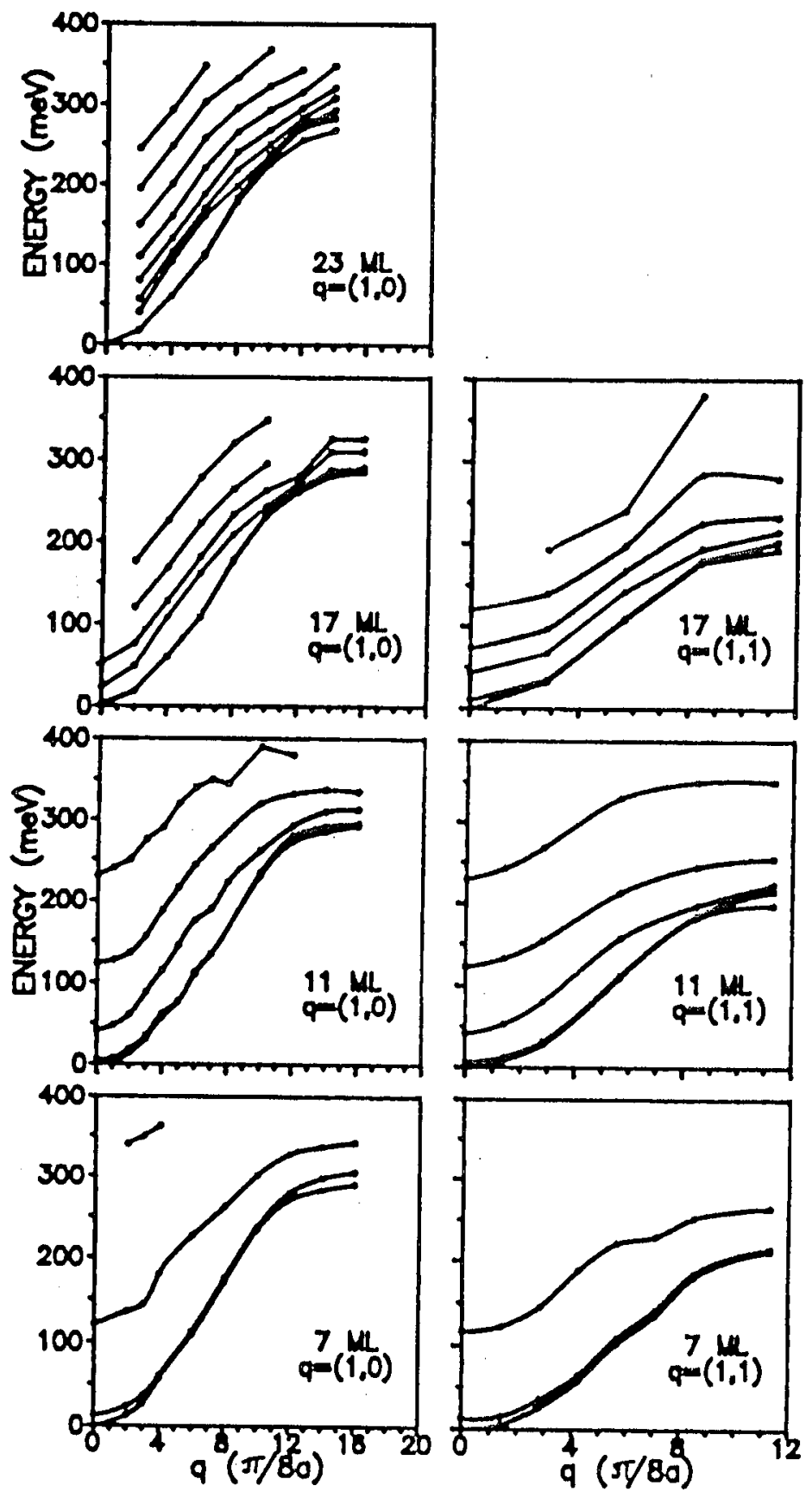

Fig. 3. The spin-wave dispersion relations for films of various tlicknesses covered with Fe. In the scale of the figures the lowest mode is degenerated or nearly degenerated. 
in Fig. 4. It can be seen that the amplitudes are large near the centre of the film, but practically equal to zero at the surfaces except for the two modes with the highest energies. Similarly as in films covered with Fe the amplitudes are almost independent of the value of the wave vector $q$. The spin-wave dispersion curves are presented in Fig. 5. The energies of the modes are considerably higher than in films covered with Fe. They are also higher than in uncovered films. The damping is quite strong. Except for low energies the spin-wave pcalss are wide and low.

One can see therefore that properties of spin waves depend strongly on a type of overlayer. They are also different in covered and uncovered films.

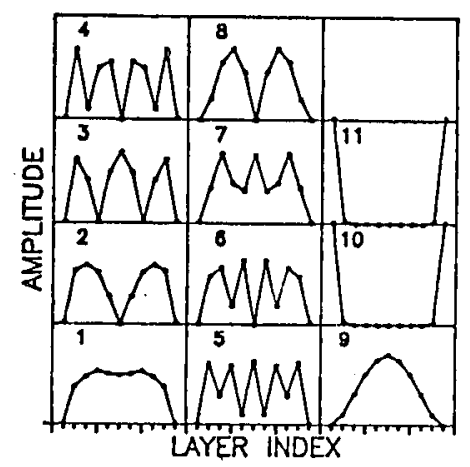

Fig. 4. The spin-wave amplitudes in $11 \mathrm{ML}$ film covered with $\mathrm{Cu}$. The wave vector $q=(1,0)$ in $\pi / 4 a$ units.
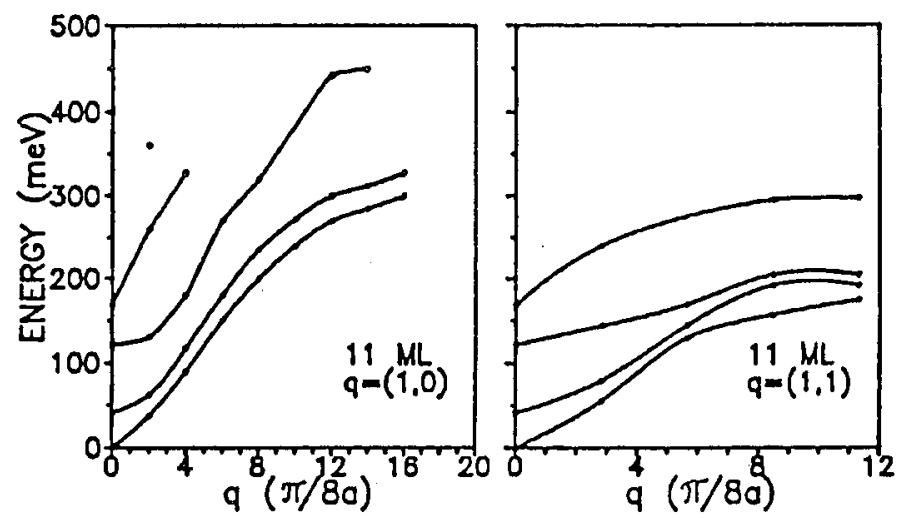

Fig. 5. The spin-wave dispersion relations for $11 \mathrm{ML}$ flim covered with $\mathrm{Cu}$.

\section{Temperature dependence of magnetization}

The temperature changes of the local magnetization $m_{\nu}$ in the layer $\nu$ are given by the following formula [2]:

$$
m_{\nu}(0)-m_{\nu}(T)=\int_{0}^{\infty} N_{\nu}(E)[\exp (E / k T)-1]^{-1} \mathrm{~d} E
$$


$N_{\nu}(E)$ denotes here the spin-wave density of states which can be expressed in terms of imaginary part of the dynamic susceptibility $\chi_{\nu \nu}(q, E)$ in the following way:

$$
N_{\nu}(E)=\frac{1}{\pi} \sum_{q} \chi_{\nu \nu}(q, E) .
$$

Therefore, the calculation of the dynamic susceptibility for various values of wave vector $q$ and various energies allows us to determine the density of states. Ilowever, in the case of films covered with $\mathrm{Fe}$ the spin-wave peaks are very narrow even for quite considerable energies. Therefore, using the formula (4) it is difficult to calculate $N_{\nu}(E)$, especially in the low-energy region, where peaks are too narrow to be well seen within the framework of the accuracy of our calculations. On the other hand, for very low values of $q$ vectors the quadratic dispersion relation is held quite well for the low lying modes. Therefore, it scems reasonable to calculate $N_{\nu}(E)$ as a sum of two contributions. In the part of the irreducible wedge of the Brillouin zone where $\operatorname{Im} \chi_{\nu \nu}(q, E)$ is well determined we use formula (4). And in the vicinity of the centre of the zone the contribution to density of states is determined according to the expression

$$
N_{\nu}(E)=\frac{1}{N} \sum_{q i}\left|A_{i \nu}\right|^{2} \delta\left(E-E_{i}(q)\right),
$$

where $E_{i}(q)=E_{0 i}+D_{i}(q) q^{2}$ is the energy of the $i$-th mode and $A_{i \nu}$ represents its amplitude in the layer $\nu$. For the lowest mode $E_{01}=0.0 \mathrm{eV}$. A slight anisotropy of the stiffness parameter $D$ is taken into account. Calculations are performed only for films consisting of $11 \mathrm{ML}$.

The calculated density of states is used in formula (3) to determine the temperature changes of the magnetization. Because of quasi-two-dimensional behaviour of density of states in the low-energy region in integral (3), the divergence at $E=0$ will appear. To avoid this, similarly as in Ref. [3] we introduce some effective field simulating the anisotropy effects. The anisotropy required for removing the divergence is of the order of error of numerical computations.

In nickel films covered with iron the temperature changes of the surface magnetization are much stronger than changes which take place inside the film. Fast decrease in the magnetization in the overlayer is a consequence of presence of the low-energy spin-wave modes which are well localized near the overlayers. The temperature dependence of the surface magnetization is quasi-linear. In the wide temperature region the surface magnetization fits the linear dependence quite well. But locally it can be fitted to a $T^{3 / 2}$ law with strongly enhanced coefficient $B_{\mathbf{s}}$. It appears that the strong surface perturbation influences the temperature dependence of the central layer magnetization in $11 \mathrm{ML}$ film. One can see (Fig. 6) that the magnetization of the central layer can be well fitted to a $T^{3 / 2}$ dependence but with two different coefficients $B_{\mathrm{c}}$. Smaller $B_{\mathrm{c}}$ is obtained in the low-temperature region (for $k T<0.1 \Delta$, where $\Delta$ represents the spin-splitting energy). Therefore, the $11 \mathrm{ML}$ film appears to be relatively thin. On the basis of calculations performed for spin waves in 17 and 23 ML films we can expect that the changes of the coefficient $B_{\mathrm{c}}$ will be smaller and they will take place at lower temperatures. 


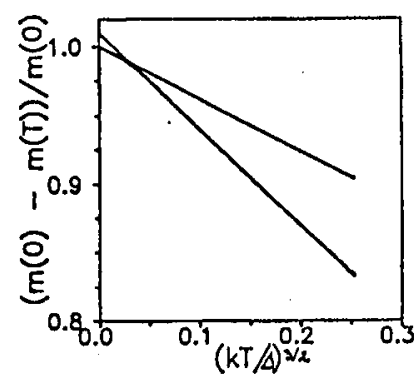

Fig. 6. Fitting of the central layer magnetization to a $T^{3 / 2}$ dependence in 11 ML film covered with Fe.

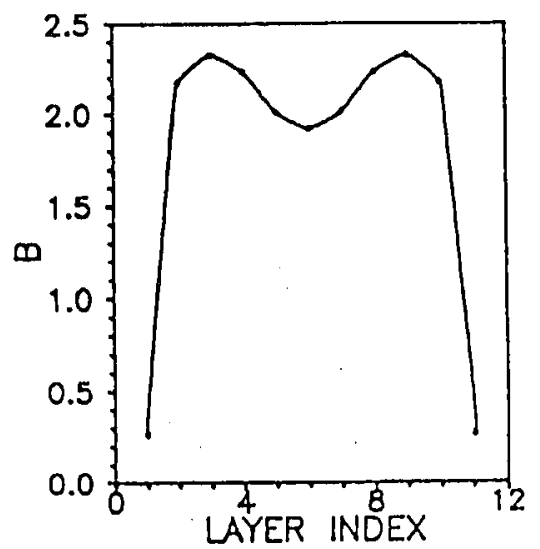

Fig. 7. The spatial distribution of the Bloch cocfficient $B$ in $11 \mathrm{ML}$ film covered with $\mathrm{Cu}$.

Therefore, in case of thicker films the temperature dependence of the central layer magnetization can be well fitted to the Bloch law. The obtained result seems to be consistent with the one obtained by Mathon and Ahmad [2]. According to Ref. [2] in the case of the considerable softening of the surface exchange the quasi-linear dependence of the surface magnetization can take place, though the magnetization curve can be locally fitted to a $T^{3 / 2}$ law. Such a strong softening is expected for the Fe overlayer on Ni substrate [18].

In $\mathrm{Cu} / \mathrm{Ni} / \mathrm{Cu}$ systems the damping of spin waves is quite considerable and the density of states can be calculated according to formula (1) for all $q$ vectors from the irreducible wedge of the Brillouin zone except for low lying modes with $q$ very close to $\Gamma$ point, where formula (5) is used. The density of states is very low in the $\mathrm{Cu}$ surface layer, but there are no considerable differences in various nickel planes. The calculated temperature dependence of the local magnetization can be fitted to a $T^{3 / 2}$ law. The Bloch coefficient $B$ slightly changes its value inside the film. The lowest value is obtained for the central layer and the highest one for the 
subinterface plane. Ilowever, the cocfficient $B$ rapidly decreases near the surface. The changes of $B$ are depicted in Fig. 7. Very small induced magnetic moment is obtained in the $\mathrm{Cu}$ overlayer and the moment decreases slowly with temperature.

\section{Conclusions}

Summarizing the results, we can state that overlayers on nickel film strongly influence the ground-state magnetic properties, as well as spin waves. The energy spectrum, amplitudes of the modes, and damping depend essentially on the surface conditions determined by the type of overlayer. As a consequence, temperature dependence of the local magnetization in the spin-wave regime is influenced. In films consisting of $11 \mathrm{ML}$ the central layer magnetization can be fitted to a $T^{3 / 2}$ law. However, the strong surface perturbation influences this relation and a quasi $T^{3 / 2}$ dependence with two different coefficients $B$ for various ranges of temperatures is obtained for films covered with Fe. The changes of the surface magnetization are strongly dependent on the surface conditions. In nickel films with free surfaces the surface magnetization fits the Bloch law quite well and the coefficient $B_{\mathrm{s}}$ is about twice as large as in the central layer [19]. When the film is covered with $\mathrm{Cu}$, the induced low magnetization is obtained for the surface layer. The coefficient $B_{\mathrm{s}}$ is also very low. But the Bloch coefficient in the interface is a little higher than the one calculated for the central layer. On the other hand, in films covered with $\mathrm{Fe}$ different behaviour of surface magnetization is observed. In iron overlayers, because of presence of strongly localized low-cnergy spin waves the decrease in the magnetization is much faster than inside the film. The temperature dependence of the surface magnetization appears to be quasi-linear.

This work was supported by the Committee for Scientific Research project No. 20250911 .

\section{References}

[1] J. Mathon, Phys. Rev. B 24, 6588 (1981).

[2] J. Mathon, S.B. Ahmad, Phys. Rev. B 37, 660 (1988).

[3] R. Świrkowicz, Physica B 107, 239 (1990).

[4] G. Bayreuther, IIyperfine Interact. 47, 237 (1989).

[5] D.T. Pierce, R.J. Celotta, J. Unguris, H.C. Siegman, Phys. Rev. B 26, 2566 (1982).

[6] M. Przybylski, J. Korecki, U. Gradmann, Appl. Phyys. A 52, 33 (1991).

[7] C. Walker, R. Droste, G. Stern, J. Tyson, J. Appl. Phys. 55, 2500 (1984).

[8] R. Świrkowicz, A. Sukiennicki, J. Magn. Magn. Mater. 104-107, 1783 (1992).

[9] R.B. Muniz, J.F. Cooke, D.M. Edwards, J. Phys. F, Metal Phys. 15, 2357 (1985).

[10] J. d'Albuquerque e Castro, D.M. Edwards, J. Mathon, R.B. Muniz, J. Magn. Magn. Mater. 93, 295 (1991); J. d'Albuquerque, private communication.

[11] H. Hasegawa, Surf. Sci. 182, 591 (1987).

[12] D.S. Wang, A.J. Freeman, II. Krakauer, Phys. Rev. B 26, 1340 (1982).

[13] R. Wu, A.J. Freeman, Phys. Rev. B 45, 7205 (1992). 
[14] J.I. Lee, S.C. Hong, A.J. Freeman, J. Magn. Magn. Maler. 104-107, 1684 (1992). [15] R. Świrkowicz, Physica B 160, 329 (1990).

[16] J. Barnas, Phys. Rev. B 45, 10427 (1992).

[17] Niu-Niu Chen, M.G. Cottam, Solid Stale Commun. 7C, 437 (1990).

[18] T. Maeda, H. Yamauchi, II. Watanabe, J. Phys. Soc. Jpn. 35, 1635 (1973).

[19] R. Świrkowicz, Acla Phys. Pol. A 80, 689 (1991). 